\title{
Abnormality Segmentation of MRI Brain Images using Fuzzy Nearest Neighbour Approach
}

\author{
T.Akhila Thankam \\ PG scollar, Dept. of Computer Science and \\ Engineering, \\ Noorul Islam Center for Higher Education \\ Kumaracoil, India.
}

\author{
K. S. Angel Viji \\ Assistant Professor, Dept. of Computer Science \\ and Engineering, \\ Noorul Islam Center for Higher Education \\ Kumaracoil, India.
}

\begin{abstract}
Many medical imaging techniques help in learning human brain. Magnetic resonance imaging, is a technique which is very efficient in differentiating between soft tissues. There are some techniques that accomplish the goal of tissues detection and extraction. The main objective is to provide a reliable tool to evaluate brain, by improving tissue contrast and visualization, thus reducing workload of specialist in the area. This allows a further systematic follow-up of the evolution of tumors or its treatment. A solution to this problem is offered by semi supervised anomaly detection after spatial normalization. It makes use of normal data modeling and then a distance measure and thresholding to determine abnormality in MRI. The estimation of the probability density function is usually used to treat every image as a network of locally coherent image partitions (overlapping blocks). So a strictly concave likelihood function for estimating abnormality onto each partition have been formulated and the local estimates are fused into a globally optimal estimate that satisfies the consistency constraints. Fuzzy based approach can be defined to enhance the performance of the system by providing active learning based approach. Fuzzy clustering algorithms proposed an energy-minimization approach to the coherent local intensity clustering (CLIC), with the aim of achieving tissue clustering of abnormalities properly. This approach provides efficient system for the easy detection of abnormalities.
\end{abstract}

\section{Keywords}

Fuzzy c-mean(FCM), MRI, Image segmentation

\section{INTRODUCTION}

IMAGE segmentation is a main task in many research fields including computer vision [3] and intelligent image and video analysis [4]. It is an essential goal to split the pixels of an image into a set of regions such that the pixels in the same region are homogeneous according to some constraint. Clustering, particularly fuzzy C-means based(FCM) clustering and its variants, have been widely used in the task of image segmentation due to their simplicity and fast convergence [3], [4], [5], [6],[7], [9]. By carefully selecting input features such as pixel color, intensity, texture, or a weighted combination of these data, the FCM algorithm can segment images to several regions in accordance with resulting clusters. FCM and other clustering-based image-segmentation approaches are improved by including the local spatial information of pixels in classical clustering procedures [2], [4], [7], [8], [9],[10]. Because of the embedded local spatial information, the new FCM has improved robustness over noises in images [2], [11], [12].With the fast advancement of MRI, which provides high resolution images, the detection of tumors is becoming increasingly more feasible [24]. However, due to their arbitrary shapes and locations, segmentation of tumor is a complex and challenging task.

Manual segmentation is thus widely employed for this purpose [25], [26], but it is very time-consuming, which may be an unrealistic approach when a large amount of data needs to be processed. But the disadvantage of manual segmentation is that the results are highly dependent on the operators expertise. So a continuous efforts have been made on automatic detection of different types of tumors in the literature. Although a great number of segmentation methods are available, such as thresholding [27], region growing [28], and clustering [29] they are problematic when applied in tumor detection. This is because tumors have similar intensities to some normal tissues. In T1-weighted MR images, a tumor is shown with intensities similar to those of cerebrospinal fluid (CSF) or graymatter(GM), so that a standard segmentation approach would group them together rather than separately. In order to solve this problem, multispectral MR images were employed for the purpose of tumor detection by many researchers. Anbeek et al. [31] presented a segmentation method for white matter(WM) tumor using five different MRI modalities including T1weighted, inversion recovery (IR), proton-densityweighted(PD), T2-weighted, and fluid attenuation inversion recovery(FLAIR). It is based on a K-nearest neighbour $(\mathrm{KNN})$ classification technique, which builds a feature space from voxel intensity and spatial information. Sajja et al. [33] used PD-weighted, T2-weighted, and FLAIR MR images to segment multiple sclerosis(MS) tumors, which involved techniques such as Parzen window classifier, morphological operations, hidden Markov random field-expectation maximization (HMRF-EM) algorithm, and fuzzy connectivity. A similar approach was employed by Datta et al. [14] to identify black holes in MS. Van Leemput et al. [15] proposed an intensity-based tissue classification algorithm implemented with T1-, T2-, and PD-weighted scans using a stochastic model for normal brain images and simultaneously detects MS tumors as outliers that are not well explained. Therefore, the development of a robust automatic tumor detection approach, which is capable of detecting tumors from a single anatomical MRI scan, is essential.

A few attempts have been made to detect brain abnormality using T1-weighted MR images only. Cuadra et al. [18] used $a$ priori models of tumor growth to segment large brain tumors. However, it is a semiautomatic approach where a seed voxel of tumors has to be chosen manually. The selection thus requires anatomical and biological knowledge of tumor growth. Stamatakis and Tyler [19] presented a statistical method to identify brain tumors. Srivastava et al. [20] employed a similar statistical approach to detect focal cortical 
dysplastic (FCD) tumors from a tumor-specific feature map constructed by computing the ratio of cortical thickness over absolute image intensity gradient. However, the disadvantage of these statistical segmentation methods is that the selection of a control group (e.g., groupsize) may have an impact on segmentation results [21]. Wei et al. [22] demonstrated that the addition of a template-driven approach to EM algorithm significantly improved the segmentation accuracy of WM abnormalities. In this paper, the conventional fuzzy c-mean (FCM) algorithm is employed for intensity-based segmentation. The spatial-location-based tissue distribution is provided by prior tissue probability maps.

In this paper section 2 deals with the methodology such as preprocessing, enhancement, clustering and segmentation techniques. Section 3 clearly shows the experimental results, performance analysis and comparison of different clustering methods and segmentation algorithms.In section 4 comparison and discussion was done. Finally this paper is concluded at section 5 .

\section{METHODOLOGY}

The total process is as follows:

1. Input MR Image.

2. Perform noise removal and contrast enhancement using filtering.

3. Also perform boundary detection.

4. Perform different color grouping with pixel intensity.

5. Apply clustering based pixel grouping. Cluster segmentation is done using two steps.

- Initialization Step

- Fuzzy Computation

6. Overlay the Segmented group.

7. Compare between clustering and segmentation algorithms. 8. Stop.

\subsection{Image acquisition}

The first stage of any vision system is the image acquisition stage. After the image has been obtained various methods of processing can be applied to the image to perform the many different vision tasks require today. However if the image has not been acquired satisfactorily then the intended tasks may not be achievable even with the aid of some form of image enhancement.

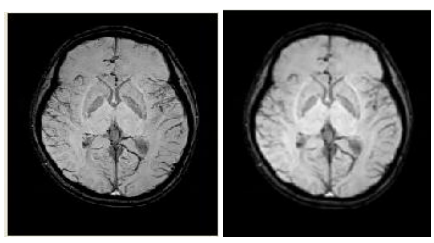

Figure 1: The sample brain MRI

\subsection{Preprocessing}

MRI brain images cannot be fed directly as the input for the FCM technique. The input image is subjected to a set of preprocessing steps so that the image gets transformed suitable for further processing. The digital image data for a spatial database requires several preprocessing procedures. The goal of digital image processing is to increase both the accuracy and the interpretability of the digital data during the image processing phase.

\subsection{Enhancement}

The image is given to enhancement stage for the removing high intensity component and the above noise. Here work is carried out to perform contrast enhancement. This part is used to enhance the smoothness towards piecewise-homogeneous region and reduce the edge blurring effects. Contrast enhancement attempts to increase the appearance of large scale light dark transitions similar to sharpening.

\subsection{Threshold based segmentation}

Thresholding is the simplest method of image segmentation. From a grayscale image, thresholding can be used to create binary images. During the thresholding process, individual pixels in an image are marked as "object" pixels if their value is greater than some threshold value (assuming an object to be brighter than the background) and as "background" pixels otherwise. This convention is known as threshold above. Variants include threshold below, which is opposite of threshold above; threshold inside, where a pixel is labeled "object" if its value is between two thresholds; and threshold outside, which is the opposite of threshold inside. Typically, an object pixel is given a value of " 1 " while a background pixel is given a value of " 0 ." Finally, a binary image is created by coloring each pixel white or black, depending on a pixel's label. The drawbacks of thresholding includes

- Threshold selection is not always straightforward.

- Pixels assigned to a single class need not form coherent regions as the spatial locations of pixels are completely ignored.

\subsection{Clustering}

A cluster is a set of nodes that communicate with each other and work toward a common goal. Fuzzy C-Means Clustering (FCM) is also known as Fuzzy ISODATA, for clustering technique. The aim of FCM is to find cluster centres (centroids) that minimize a dissimilarity function. The fuzzy c-means algorithm, Zang et al.,[12] allows each data point to belong to a cluster to a degree specified by a membership grade, and thus each point may belong to several clusters. The FCM employs fuzzy partitioning such that a data point can belong to all groups with different membership grades between 0 and 1. FCM is an iterative algorithm and it is a method of grouping the similar types of pixels in the image. Fuzzy c-means is different from hard c-means, mainly because it employs fuzzy partitioning, where a point can belong to several clusters with degrees of membership. Clustering of numerical data forms the basis of many segmentation and system modeling algorithms. The purpose of clustering is to identify natural groupings of data from a large data set to produce a concise representation of a system's behavior. 
Fuzzy c-means (FCM) is a data clustering technique wherein each data point belongs to a cluster to some degree that is specified by a membership grade. This technique was originally introduced by H.Yan et al.[29] as an improvement on earlier clustering methods. It provides a method that shows how to group data points that populate some multidimensional space into a specific number of different clusters.FCM starts with an initial guess for the cluster centers, which are intended to mark the mean location of each cluster. The initial guess for these cluster centers is most likely incorrect. Additionally, FCM assigns every data point a membership grade for each cluster. By iteratively updating the cluster centers and the membership grades for each data point, FCM iteratively moves the cluster centers to the right location within a data set. This iteration is based on minimizing an objective function that represents the distance from any given data point to a cluster center weighted by that data point's membership grade. By using information returned by FCM to represent the fuzzy qualities of each cluster.

A new cluster validity index is proposed that determines the optimal partition and optimal number of clusters for fuzzy partitions obtained from the fuzzy $c$-means algorithm. The proposed validity index exploits an overlap measure and a separation measure between clusters. The overlap measure, which indicates the degree of overlap between fuzzy clusters, is obtained by computing an inter-cluster overlap. The separation measure, which indicates the isolation distance between fuzzy clusters, is obtained by computing a distance between fuzzy clusters.A good fuzzy partition is expected to have a low degree of overlap and a larger separation distance. Fuzzy cluster-validity criterion tends to evaluate the quality of fuzzy $c$-partitions produced by fuzzy clustering algorithms. Many functions have been proposed. Some methods use only the properties of fuzzy membership degrees to evaluate partitions. Others techniques combine the properties of membership degrees and the structure of data. Major problems exist in both crisp and fuzzy clustering algorithms. The fuzzy $c$-means type of algorithms use weights determined by a power $m$ of inverse distances that remains fixed over all iterations and over all clusters, even though smaller clusters should have a larger.

A method uses a different "distance" for each cluster that changes over the early iterations to fit the clusters. Clustering refers to the process of unsupervised partitioning of a data set based on a dissimilarity measure, which determines the cluster shape. Considering that cluster shapes may change from one cluster to another, it would be of the utmost importance to extract the dissimilarity measure directly from the data by means of a data model.

The fuzzy c-means (FCM) clustering algorithm has been extensively used for pattern recognition. It has also been used in the process of generating fuzzy rules from data. It has been used with success in the soft segmentation of MR images and for the estimation of partial volumes.FCM employs fuzzy partitioning such that a given data point can belong to several groups with the degree of belongingness specified by membership grades between 0 and 1.The FCM algorithm is simply an iterative procedure. In a batch mode operation FCM determines the cluster centers $\mathrm{Ci}$ and the membership matrix $\mathrm{U}$ using following steps.
Step 1: Initialize the cluster centers the membership matrix $U$ with random values between 0 and 1 such that the

following constraints are satisfied. $\quad \sum_{j=1}^{c} u_{j k}=1$

Step 2: Calculate ' $\mathrm{C}$ ' fuzzy cluster centers $\mathrm{Ci}, \mathrm{i}=1,2, \ldots \ldots . ., \mathrm{C}$

$$
\begin{aligned}
\text { Step } 3: \quad \text { Compute the cost functions } \\
J_{m}(U, Y)=\sum_{k=1}^{n} \sum_{j=1}^{c}\left(u_{j k}\right)^{n} E_{j}\left(x_{k}\right)
\end{aligned}
$$

Where,

$Y=\left\{y_{i}\right\}$,is the set of centers of clusters.

$\mathrm{E}_{\mathrm{j}}\left(\mathrm{x}_{\mathrm{k}}\right)$, is a dissimilarity measure between the sample $\mathrm{x}_{\mathrm{k}}$ and the center $\mathrm{y}_{\mathrm{j}}$ of a specific cluster $\mathrm{j}$.

$\mathrm{U}=\left[\mathrm{u}_{\mathrm{jk}}\right]$, is the $\mathrm{c} \times \mathrm{n}$ fuzzy c-partition matrix, containing the membership values of all samples in all clusters.

$\mathrm{m} \in(1, \infty)$, is a control parameter of fuzziness.

Stop if either $\mathrm{J}_{\mathrm{m}}$ below a certain tolerance or it is improved over previous iteration.

Step 4: Compute a new $U$ and repeat the steps until an optimum result is obtained.

Abnormality segmentation of MRI Brain Images proposes a neighbour that uses fuzzy rough set theory to improve the Fuzzy nearest neighbor(FNN) approach. It was shown that previous attempts to use fuzzy rough set theory to improve the Fuzzy nearest neighbour algorithm have some shortcomings and we overcome them by using the fuzzy positive region to measure the quality of the nearest neighbours in the fuzzy nearest neighbour approach. FNN approach is improved by weighting the nearest neighbour instances according to their membership degree to the fuzzy positive region. Fuzzy based approach can be defined to enhance the performance of the system by providing active learning based approach. Fuzzy clustering algorithms proposed an energy-minimization approach to the coherent local intensity clustering with the aim of achieving tissue classification and clustering of abnormalities properly. FNN approach provides efficient system for the easy detection of abnormalities. FNN will assign a higher weight in the voting strategy to the four instances of the minority class and the target instance will be assigned to the minority class.

\section{EXPERIMENTAL RESULTS}

Experiment was conducted for five patients and two results are shown below in Figure 2. 


\begin{tabular}{|c|c|c|}
\hline Process & Tumor 1 & Tumor 2 \\
\hline Input image & & \\
\hline Preprocessing & & \\
\hline $\begin{array}{c}\text { Fuzzy Clustering(Fuzzy nearest neighbor } \\
\text { approach) }\end{array}$ &
\end{tabular}

Figure 2: Clustering process for two patients

The overlay of the segmented image is shown in Figure 3.

\begin{tabular}{|c|c|c|c|}
\hline Process & $\begin{array}{c}\text { Various Channel } \\
\text { Information1t }\end{array}$ & $\begin{array}{c}\text { Various Channel } \\
\text { Information2t }\end{array}$ & $\begin{array}{c}\text { Various Channel } \\
\text { Information3t }\end{array}$ \\
\hline Feature/Segmentation & & \\
\hline
\end{tabular}

Figure3 : Feature/Segmentation Process

\subsection{Performance Analysis}

Sensitivity and accuracy are statistical measures of the performance of a binary classification test. Sensitivity is defined as actual positives which are correctly identified as positive. The success or failure rate of the proposed algorithm is measured using accuracy. For finding sensitivity and accuracy, it is necessary to compute some of the terms like, True positive (TP), True negative (TN), False negative (FN) and False positive (FP). The clustering result is calculated with the help of the following data. The following metrics, are used to detect the clustering efficiency.

Sensitivity $=\mathrm{TP} /(\mathrm{TP}+\mathrm{FN}) * 100 \%$

Accuracy $=(\mathrm{TN}+\mathrm{TP}) /(\mathrm{TN}+\mathrm{TP}+\mathrm{FN}+\mathrm{FP}) * 100 \%$

The ground truth (GT), segmented image (SI), TP, TN, FP and FN values are calculated first. From those values completeness, correctness and accuracy measures are calculated as per the above formula for various segmentation and clustering algorithms. The ground truth region and segmented region of the tumor are shown in figure 4 .

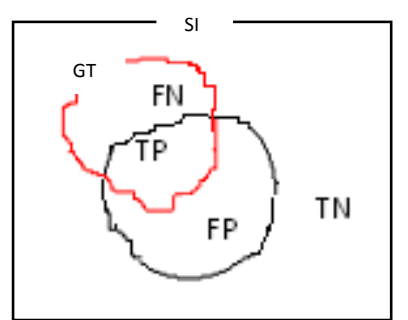

Figure 4: Ground truth and segmented tumor region

The input image, ground truth region, segmented region and clustered region(fuzzy nearest neighbour) of the tumor are shown in the figure 5 . The sensitivity and accuracy were calculated for the above segmented images and the values are shown in table 1 and 2. 


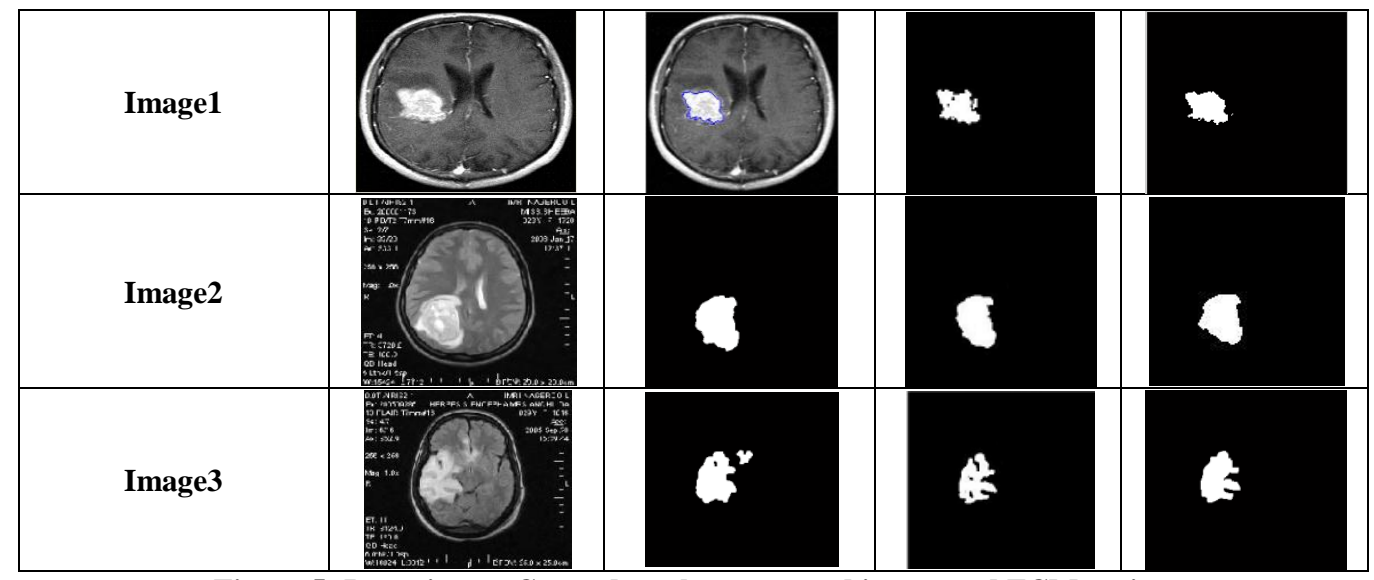

Figure 5: Input image,Ground truth, segmented image and FCM region

Table 1: Sensitivity

\begin{tabular}{|c|c|c|}
\hline Images & Threshold based segmentation & Fuzzy C-means Clustering \\
\hline Image 1 & $77.3 \%$ & $86 \%$ \\
\hline Image 2 & $76.9 \%$ & $85.4 \%$ \\
\hline Image 3 & $77.3 \%$ & $84.9 \%$ \\
\hline
\end{tabular}

Table 2:Accuracy

\begin{tabular}{|c|c|c|}
\hline Images & Threshold based segmentation & Fuzzy C-means Clustering \\
\hline Image 1 & $75.6 \%$ & $88.5 \%$ \\
\hline Image 2 & $76.7 \%$ & $87.6 \%$ \\
\hline Image 3 & $78.2 \%$ & $87.3 \%$ \\
\hline
\end{tabular}

\section{COMPARISON AND DISSCUSSION}

Among the two methods threshold based method have the lowest measure of sensitivity than FCM method. Accuracy is also low in threshold based method compared with Fuzzy C
Mean method. The comparison chart is shown in Figure 5a and Figure $5 b$.

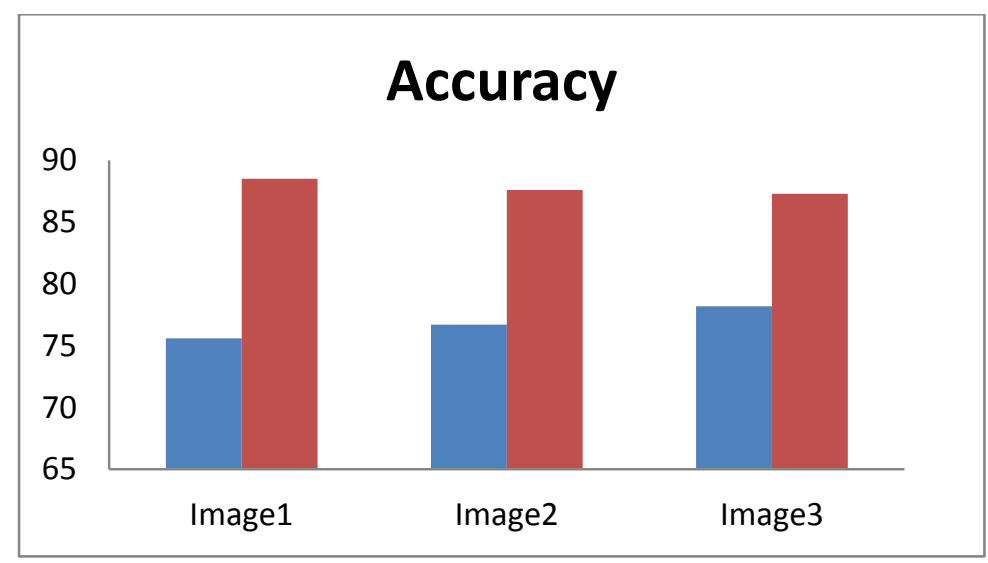

Figure 5a: Accuracy 


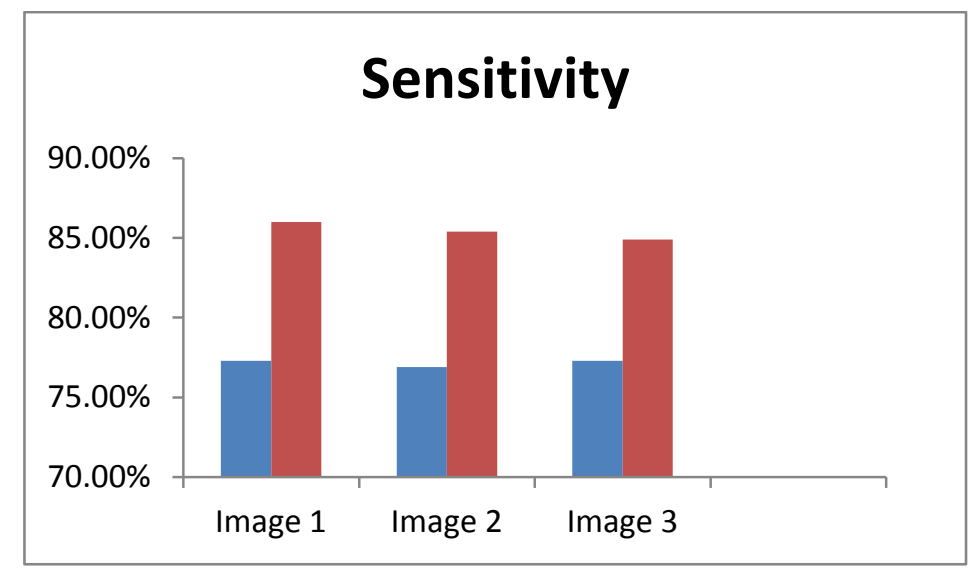

Figure 5b: Sensitivity

\section{CONCLUSION}

Image partitioning combined with a distributed estimation algorithm to deal with the high dimensional problem of statistical modelling. The constructed statistical model of normal brain images has been applied to the segmentation of brain pathologies. The method based on ROC analysis demonstrated segmentation improvement over un-invariants statistics. Two-group analysis performed with SPM specifies semi supervised learning. The FCM algorithm can largely overcome the difficulties raised by noise, low contrast, and bias fields, and is capable of producing more accurate results than several state-of-the-art algorithms. Experimental results on this database show that the FCM method can segment the tumor accurately than threshold based method.

\section{REFERENCES}

[1] Evangelia,I. "Abnormality Segmentation in Brain Images Via Distributed Estimation". IEEE Transactions On Information Technology In Biomedical. Vol.16, May 2012,no.3.

[2] W. L. Cai, S. C. Chen, and D. Q. Zhang. "Fast and robust fuzzy c-means clustering algorithms incorporating local information for image segmentation", Pattern Recognit. vol. 40, Mar 2007,no. 3, pp. 825-838.

[3] S. Dambreville, Y. Rathi, and A. Tannenbaum, "A framework for image segmentation using shape models and kernel space shape priors,"IEEE Trans. Pattern Anal. Mach. Intell., vol. 30, Aug 2008, no. 8, pp. $1385-1399$.

[4] K.Sikka, N. Sinha, P. K. Singh, and A. K. Mishra, "A fully automated algorithm undermodified FCM framework for improved brain MR image segmentation," Magn. Reson. Imaging, vol. 27, Sep 2009, no. 7, pp. 994-1004.

[5] M. S. Yang and H. S. Tsai, "A Gaussian kernel-based fuzzy c-means algorithm with a spatial bias correction," Pattern Recognit. Lett., vol. 29, Sep. 1, 2008, no. 12, pp. 1713-1725.

[6] R. J. He, S. Datta, B. R. Sajja, and P. A. Narayana, "Generalized fuzzy clustering for segmentation of multi-spectral magnetic resonance images," Comput.
Med. Imaging Graph., vol. 32, Jul 2008, no. 5, pp. $353-$ 366.

[7] Y. A. Tolias and S. M. Panas, "Image segmentation by a fuzzy clustering algorithm using adaptive spatially constrained membership functions,"IEEE Trans. Syst., Man, Cybern. A, Syst., Humans, vol. 28, May 1998, no. 3, pp. 359-369.

[8] Y. Xia, D. G. Feng, T. J. Wang, R. C. Zhao, and Y. N. Zhang, "Image segmentation by clustering of spatial patterns," Pattern Recognit. Lett.,vol. 28, Sep. 1,2007,no. 12, pp. $1548-1555$

[9]J. Z.Wang, J. Kong, Y. H. Lu, M. Qi, and B. X. Zhang, "A modified FCM algorithm for MRI brain image segmentation using both local and nonlocal spatial constraints," Comput. Med. Imaging Graph., vol. 32, Dec. 2008,no. 8 ,

pp. 685-698.

[10]L. L. He and I. R. Greenshields, "AnMRF spatial fuzzy clustering method for fMRI SPMs," Biomed. Signal Process. Control, vol. 3,Oct 2008, no. 4, pp. 327-333.

[11]D. Q. Zhang and S. C. Chen, "A novel kernelized fuzzy C-means algorithm with application in medical image segmentation," Artif. Intell. Med., vol. 32 , Sep 2004 , no. 1 , pp. 37-50.

[12]S. C. Chen and D. Q. Zhang, "Robust image segmentation using FCM with spatial constraints based on new kernel-induced distance measure,"IEEE Trans. Syst., Man, Cybern. B, Cybern., vol. 34, Aug 2004 no. 4, pp. 1907-1916.

[13] B.Yuan, G. J. Klir, and J. F. Swan-Stone, "Evolutionary fuzzy c-means clustering algorithm," in Proc. 1995 IEEE Int. Conf. Fuzzy Syst. Int. Joint Conf. 4th IEEE Int. Conf. Fuzzy Syst. 2nd Int. Fuzzy Eng. Symp., pp. 663-670.

[14] S. Datta, B. R. Sajja, R. He, J. S. Wolinsky, R. K. Gupta, and P. A. Narayana, "Segmentation and quantification of black holes in multiple sclerosis," NeuroImage, vol. 29,Sep 2006, pp. 467-474.

[15]K.VanLeemput,F.Maes,D.Vandermeulen,A.Colchester,an dP.Suetens, "Automated segmentation of multiple sclerosis lesions by model outlier detection," IEEE 
Trans. Med. Imag., vol. 20, Aug.2001, no. 8, pp. 677688

[16] F. B. Mohamed, S. Vinitski, C. F. Gonzalez, S. H. Faro, F. A. Lublin,R. Knobler, and J. E. Gutierrez, "Increased differentiation of intracranial white matter lesions by multispectral 3D-tissue segmentation: Preliminary results," Magn. Reson. Imag., vol. 19,Sep 2001, pp. 207-218.

[17] F. Yang, T. Jiang, W. Zhu, and F. Kruggel, "White matter lesion segmentation from volumetric MR images," Lect. Notes Comput. Sci., vol. 3150,Dec 2004, pp. 113-120.

[18] M. B. Cuadra, C. Pollo, A. Bardera, O. Cuisenaire, J.-G. Villemure, and J.-P. Thiran, "Atlas-based segmentation of pathological MR brain images using a model of lesion growth," IEEE Trans.Med. Imag., vol. 23, no. 10,pp. $1301-1314$,

[19] E. A. Stamatakis and L. K. Tyler, "Identifying lesions on structural brain images - Validation of the method and application to neuropsychological patients," Brain Lang., vol. 94, Oct. 2004, pp. 167-177.

[20] S. Srivastava, F. Maes, D. Vandermeulen, W. V. Paesschen, P. Dupont,and P. Suetens, "Feature-based statistical analysis of structural MR data for automatic detection of focal cortical dysplastic lesions," NeuroImage, vol. 27, July 2005, pp. 253-266.

[21] S. Shen, A. J. Szameitat, and A. Sterr, "VBM lesion detection depends on the normalization template: A study using simulated atrophy," Magn.Reson. Imag., vol. 25, pp. 1385-1396, 2007.540 IEEE TRANSACTIONS ON INFORMATION TECHNOLOGY IN BIOMEDICINE, VOL. 12, JULY 2008, NO. 4.

[22] X. Wei, S. K. Warfield, K. H. Zou, Y. Wu, X. Li, A. Guimond, J. P.Mugler, III, R. R. Benson, L. Wolfson, H. L. Weiner, and C. R. G.Guttmann, "Quantitative analysis of MRI signal abnormalities of brain white matter with high reproducibility and accuracy," J. Magn. Reson.Imag., vol. 15,2002, pp. 203-209.

[23] M. Kamber, R. Shinghal, D. L. Collins, G. S. Francis, and A. C. Evans,"Model-based 3D segmentation of multiple sclerosis lesions in magnetic resonance brainimages,"IEEETrans.Med. Imag., vol. 14, Sep. 1995,no. 3, pp. 442-453.
[24] T. Ogawa, A. Inugami, H. Fujita, J. Hatazawa, E. Shimosegawa,K. Noguchi, T. Okudera, I. Kanno, K. Uemura, and A. Suzuki, "MR diagnosis of subacute and chronic subarachnoid hemorrhage: Comparison with CT," Amer. J. Roentgenol., vol. 165,1995, pp. 1257 1262.

[25] R. Adolphs, H. Damasio, D. Tranel, G. Cooper, and A. R. Damasio, "Arole for somatosensory cortices in the visual recognition of emotion as revealed by threedimensional lesion mapping," J. Neurosci., vol. 20,2000,pp. 2683-2690.

[26] E. Bates, S. M. Wilson, A. P. Saygin, F. Dick, M. I. Sereno, R. T. Knight,and N. F. Dronkers, "Voxel-based lesion-symptom mapping," NatureNeurosci., vol. 6, 2003,pp. 448-450.

[27] G. H. L. Lemieux, K. Krakow, and F. G. Woermann, "Fast, accurate, and reproducible automatic segmentation of the brain in weighted volume MRI data," Magn. Reson. Med., vol. 42,1999,pp. 127-135.

[28] H. Tang, E. X.Wu, Q. Y. Ma, D. Gallagher, G. M. Perera, and T. Zhuang,"MRI brain image segmentation by multi-resolution edge detection and region selection," Comput. Med. Imag. Graph., vol. 24, 2000,pp. 349-357.

[29] A.W. C. Liew and H. Yan, "An adaptive spatial fuzzy clustering algorithm for 3-D MRimage segmentation," IEEE Trans. Med. Imag., vol. 22, Sep. 2003, no. 9, pp. 1063-1075.

[30] J. Ashburner and K. J. Friston, "Unified segmentation," NeuroImage,vol. 26,2005, pp. 83851.

[31] P. Anbeek, K. L. Vincken, M. J. P. van Osch, R. H. C. Bisschops, and J. Van Der Grond, "Automatic segmentation of different-sized white matter lesions by voxel probability estimation," Med. Image Anal., vol. 8,2004,pp. 205-215.

[32] P. Anbeek, K. L. Vincken, G. S. van Bochove,M. J. P. van Osch, and J. Van Der Grond, "Probabilistic segmentation of brain tissue in MR imaging,"NeuroImage, vol. 27,2005, pp. 795-804.

[33] B. R. Sajja, S. Datta, R. He, M. Mehta, R. K. Gupta, J. S. Wolinsky, and P. A. Narayana, "Unified approach for multiple sclerosis lesion segmentation on brain MRI," Ann. Biomed. Eng., vol. 34,Oct 2006, pp. 142-151. 